\title{
Chemical composition of the extracellular slime glycolipoprotein of Pseudomonas aeruginosa and its relation to gentamicin resistance
}

\author{
G. ARSENIS and G. DIMITRACOPOULOS*
}

\section{Department of Microbiology, National University of Athens, and "Department of Microbiology, School of Medicine, University of Patras, Greece}

\begin{abstract}
Summary. The slime glycolipoproteins (GLPs) extracted from Pseudomonas aeruginosa strain $\mathrm{C} 2$ and its laboratory-induced gentamicin-resistant variant were analysed for gross chemical composition. The GLP of the wild-type strain contained significantly greater amounts of neutral sugars, uronic acid and thiobarbituric-reactive material $(p<0.001)$ than the GLP of the gentamicin-resistant variant. Also significantly higher $(\mathrm{p}<0.01)$ was the amino-sugar content of the GLP from the wild-type strain. Paper chromatographic analyses of the hydrolysates of the GLPs revealed that two neutral sugars, rhamnose and mannose, were absent from the GLP of the resistant variant. The GLP of strain C2 contained significantly less protein than the GLP of the gentamicin-resistant variant.
\end{abstract}

\section{Introduction}

The more general use of the aminoglycoside antibiotic gentamicin against Pseudomonas aeruginosa has led to an observed increase in resistance and several lines of research point towards the envelope of $P$. aeruginosa having a significant role in resistance by an exclusion mechanism (Sykes and Morris, 1975). Many of the clinical gentamicinresistant isolates of $P$. aeruginosa do not contain known forms of gentamicin-modifying enzymes and show a reduced permeability to gentamicin (Bryan and Van Den Elzen, 1977). It has also been reported that the amount of surface lipids in laboratory-induced resistant variants of $P$. aeruginosa is greater than that of the parent sensitive strains (Pechey and James, 1973).

The production of extracellular slime is considered to be characteristic of $P$. aeruginosa (Haynes, 1951) and has been studied as a potential virulence factor (Sensakovic and Bartell, 1974; Dimitracopoulos and Bartell, 1980). It has even been proposed that the slime is responsible for the high degree of resistance of $P$. aeruginosa to antibiotics (Brown and Richards, 1964).

In a previous paper, evidence was presented for the existence of two additional protein bands at isoelectric points of about $p \mathrm{H} 5.62$ and $p \mathrm{H} 5.3$ in the glycolipoprotein (GLP) fraction of the extracellular slime layer of a laboratory-induced gentamicinresistant variant of $P$. aeruginosa (Lambris et al.,

Received 4 Jan. 1985; accepted 7 May 1985
1980). In this paper we report quantitative and qualitative differences in the chemical composition of GLP between the wild type and a laboratoryinduced gentamicin-resistant variant of $P$. aeruginosa.

\section{Materials and methods}

\section{Bacterial strains}

Pseudomonas aeruginosa strain C2 was isolated from a clinical specimen and the minimal inhibitory concentration (MIC) of gentamicin was $0.4 \mathrm{mg} / \mathrm{L}$. A laboratory resistant variant grown in gentamicin $250 \mathrm{mg} / \mathrm{L}$ was produced from the sensitive wild strain by repeated passage in Trypticase Soy Broth (BBL Microbiology Systems, Cockeysville, MD, USA) containing increasing concentrations of gentamicin (Dimitracopoulos et al., 1979). The resistant variant differed from the wild strain in lytic phage pattern, phage adsorption and agglutination with the seven Fisher's antisera. Reversion to the original properties of the wild strain was demonstrated after ten serial subcultures in antibiotic-free broth (Dimitracopoulos et al., 1979).

\section{Extraction and purification of slime GLP}

The GLP fraction was obtained from the extracellular slime layer of $P$. aeruginosa strain $\mathrm{C} 2$ and its resistant variant (Bartell et al., 1970; Sensakovic and Bartell, 1974). Slime was extracted in $0 \cdot 15 \mathrm{M} \mathrm{NaCl}$ from bacterial cultures grown for $18 \mathrm{~h}$ on sheets of cellophane overlying Trypticase Soy Agar (BBL). The extract was precipitated with ethanol, clarified by centrifugation at $16300 \mathrm{~g}$ for 30 
min, and dialysed overnight against water. The dialysate was centrifuged at $105000 \mathrm{~g}$ for $3 \mathrm{~h}$ and the supernatant fluid containing the GLP fraction was lyophilised. Column chromatography was performed with both Sephadex G-100 and G-200 (Pharmacia Fine Chemicals, Fullerton, CA, USA) and elution was affected with $5 \mathrm{~mm}$ Tris buffer $p \mathrm{H}$ 7.2. Anion exchange chromatography was performed on diethylaminoethyl (DEAE) cellulose ( $\mathrm{Cel}$ lex D, BioRad Laboratories, Richmond, CA, USA) in Tris- $\mathrm{HCl}$ buffer $(0.05 \mathrm{~m}$ Tris, $p \mathrm{H} \mathrm{7.2)}$ and elution was accomplished with a continuous gradient of $\mathrm{KCl}$ from 0.3 to $1.0 \mathrm{M}$. All eluates were monitored for protein and carbohydrate. Cellulose acetate electrophoresis was performed on Sepraphore II strips (Gelman Instrument Co., Ann Arbor, MC, USA) in sodium barbital- $\mathrm{HCl}$ buffer,

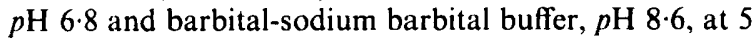
$\mathrm{mA} /$ strip for $60 \mathrm{~min}$ followed by staining with Ponceau $\mathrm{S}$ for protein and periodic acid Schiff's reagent for carbohydrate. Immunodiffusion was performed in Special Noble Agar (Difco) $1.5 \%$ in barbital-sodium barbital buffer (veronal buffer), $p \mathrm{H} 8.6$, overnight at room temperature. The antiserum used was prepared in rabbits by injecting purified GLP as previously described (Bartell et al., 1970). Immuno-electrophoresis was performed on glass slides overlaid with Special Noble Agar $2.0 \%$ in veronal buffer, $p \mathrm{H} 8.6$, at $5 \mathrm{~mA} /$ slide for $60 \mathrm{~min}$ at $4^{\circ} \mathrm{C}$. Ultracentrifugation of GLP was performed at a concentration of $10 \mathrm{mg} / \mathrm{ml}$ in $0.2 \mathrm{M} \mathrm{NaCl}$ at $20^{\circ} \mathrm{C}$. The sedimentation pattern was visualised by Schlieren optics.

\section{Chemical analysis}

The chemical composition of the GLPs was determined by standard methods and average values are presented based on six determinations of each component. Total neutral sugars were determined by the anthrone reaction (Spiro, 1966), total amino-sugars by the method of Belcher et al. (1954), protein by the method of Lowry et al., (1951), uronic acid by the method of Bitter and Muir (1962), thiobarbituric-reactive material by the method of Osborn (1963) and Ellwood (1970), and phosphorus by the method of Chen et al. (1956). For the identification of amino sugars, the GLP fractions were hydrolysed in $6 \mathrm{~N}$ $\mathrm{HCl}$ at $100^{\circ} \mathrm{C}$ for $24 \mathrm{~h}$. The hydrolysates were developed in butanol-pyridine-distilled water $(6: 4: 3)$ on descending paper chromatograms and the separated amino-sugars were visualised with acetylacetone and $p$-dimethylaminobenzaldehyde. For the identification of neutral sugars, the GLPs were hydrolysed in $6 \mathrm{~N} \mathrm{HCl}$ at $100^{\circ} \mathrm{C}$ for $24 \mathrm{~h}$. The hydrolysates were developed in a mixture of butanolethanol-distilled water $(4: 1: 2)$ containing $1 \% \mathrm{v} / \mathrm{v}$ of ammonia solution $25 \%$ on descending paper chromatograms and the separated neutral sugars were visualised with $\mathrm{HCl}-p$-anisidine.

\section{Statistical analysis}

The significance of differences in the chemical composition of GLP between the wild and the laboratory-induced gentamicin-resistant variant was determined by the twotailed $t$ test.

\section{Results}

The glycolipoproteins extracted from the slime layer of $P$. aeruginosa $\mathrm{C} 2$ and its gentamicinresistant variant were analysed for gross chemical composition and the results, which are shown in table I, represent mean values of six determinations of each component on one lot of each GLP. Compared with that of the gentamicin-resistant variant, the GLP of the wild-type strain C2 contained significantly greater amounts of neutral sugars, uronic acid and thiobarbituric-reactive

Table I. Chemical composition of extracellular slime GLPs derived from $P$. aeruginosa $\mathrm{C} 2$ and its gentamicin-resistant variant grown in gentamicin $250 \mathrm{mg} / \mathrm{L}$

\begin{tabular}{|c|c|c|c|c|}
\hline \multirow[b]{2}{*}{ Component } & \multicolumn{2}{|c|}{$\begin{array}{l}\text { Percentage (mean } \pm \text { SEM })^{*} \text { of dry } \\
\text { weight of slime GLP in }\end{array}$} & \multirow[b]{2}{*}{$t$ Value } & \multirow[b]{2}{*}{$\mathrm{p}$} \\
\hline & P. aeruginosa $\mathrm{C} 2$ & $\begin{array}{l}\text { gentamicin- } \\
\text { resistant variant }\end{array}$ & & \\
\hline Total neutral sugars & $22 \cdot 33 \pm 0.38$ & $15 \cdot 50 \pm 0.56$ & +10.06 & $<0.001$ \\
\hline Total amino sugars & $11.48 \pm 0.75$ & $8 \cdot 30 \pm 0.44$ & +3.62 & $<0.01$ \\
\hline Uronic acid & $6.50 \pm 0.22$ & $4.00 \pm 0.20$ & $+8 \cdot 20$ & $<0.001$ \\
\hline Thiobarbituric- & & & & \\
\hline $\begin{array}{l}\text { reactive material } \\
\text { Protein }\end{array}$ & $\begin{array}{r}0.93 \pm 0.02 \\
20.80 \pm 0.24\end{array}$ & $\begin{array}{r}0.68 \pm 0.01 \\
22.40 \pm 0.21\end{array}$ & $\begin{array}{l}+9 \cdot 26 \\
-4 \cdot 91\end{array}$ & $\begin{array}{l}<0.001 \\
<0.001\end{array}$ \\
\hline Phosphorus & $0.67 \pm 0.01$ & $0.70 \pm 0.02$ & -0.97 & $>0.1$ \\
\hline
\end{tabular}

\footnotetext{
*Results of six determinations of each component on one batch of each
} GLP. 
Table II. Paper chromatographic analysis of the neutral sugars of slime GLPs derived from $P$. aeruginosa $\mathrm{C} 2$ and its variant grown in gentamicin $250 \mathrm{mg} / \mathrm{L}$

\begin{tabular}{|c|c|c|c|c|}
\hline \multirow[b]{2}{*}{ Source of GLP } & \multicolumn{4}{|c|}{ Presence $(+)$ or absence $(-)$ of } \\
\hline & glucose & rhamnose & mannose & galactose \\
\hline P. aeruginosa $\mathrm{C} 2$ & + & + & + & + \\
\hline Gentamicin-resistant variant & + & - & - & + \\
\hline
\end{tabular}

material $(p<0.001)$. The amino-sugar content of GLP derived from the wild-type strain C2 was also significantly higher than that of GLP derived from the gentamicin-resistant variant $(p<0.01)$. In contrast, the GLP of the wild-type strain C2 contained significantly less protein than the GLP of the gentamicin-resistant variant (mean values 20.80 and $22 \cdot 40$, respectively). No significant difference was found between the phosphorus contents (mean values 0.67 and 0.70 ) of the two strains.

The differences observed in the neutral and amino-sugar content of the GLPs led us to investigate these components further. Paper chromatographic analyses revealed four neutral sugars in GLP hydrolysate of strain C2 and two in a similar preparation of its gentamicin-resistant variant (table II). The four neutral sugars of strain $\mathrm{C} 2$ were glucose, rhamnose, mannose and galactose, and those of its resistant variant were glucose and galactose. The paper chromatographic analyses revealed the presence of two amino-sugars in each GLP; these were identified as glucosamine and galactosamine.

\section{Discussion}

The data presented indicate that the laboratoryinduced resistance of $P$. aeruginosa to gentamicin is accompanied by quantitative and qualitative changes in the chemical composition of slime GLP.

In a previous report (Lambris et al., 1980) isoelectric focusing of the slime GLP of strain C2 showed four protein bands within the $p \mathrm{H}$ range 5.46-5.6; two further bands at about $p \mathrm{H} 5.62$ and $p \mathrm{H} 5.3$ appeared in the slime GLP of the variant grown in the presence of gentamicin $250 \mathrm{mg} / \mathrm{L}$. The results of the present study are consistent with these findings, the GLP of wild-type strain C2 containing significantly less protein than the GLP of the gentamicin-resistant variant $(p<0.001)$. Taken together, these results suggest that laboratoryinduced resistance to gentamicin effects both quan- titative and qualitative changes in the protein component of slime GLP.

Moreover our observations indicate that laboratory-induced resistance to gentamicin caused quantitative alterations in other components of slime GLP, namely neutral sugars, uronic acid, thiobarbituric-reactive material and amino-sugars. The amount of these components derived from the resistant variant was significantly lower than that from the wild-type strain. Paper chromatographic analyses revealed that the GLP of the resistant variant lacked two neutral sugars, but no qualitative differences were found in the amino-sugar content of the two strains.

Slime GLP by its inactivation of phages, is seen to possess receptor-like properties (Bartell et al., 1971; Reese et al., 1974). The significant reduction in the amount of neutral and amino-sugars of the GLP from our resistant variant may explain the inability of the cells to adsorb the phages that were active against the parent strain, and the absence of rhamnose and mannose may indicate the functional importance of these neutral sugars in phage-adsorption.

Besides possessing receptor-like properties, the slime GLP of $P$. aeruginosa seems to contribute to the pathogenesis of infection with the organism (Sensakovic and Bartell, 1974; Lynn et al., 1977; Dimitracopoulos and Bartell, 1980), has antiphagocytic activity, exerts a mitogenic effect on human peripheral blood and cord blood lymphocytes and activates human complement via the alternative pathway (Papamichail et al., 1980; Lambris et al., 1982). Lysogenisation of $P$. aeruginosa can result in considerable changes in the chemical composition and antigenicity of the slime GLP and, perhaps more significantly, in the pathogenicity of the cell (Dimitracopoulos and Bartell, 1979). The extensive alterations in the surface of the $P$. aeruginosa cell described in this communication may also have implications in the biological activities of slime GLP, but such a relationship remains to be clarified. 


\section{REFERENCES}

Bartell P F, Orr T E, Chudio B 1970 Purification and chemical composition of the protective slime antigen of Pseudomonas aeruginosa. Infection and Immunity 2:543-548.

Bartell P F, Orr T E, Reese J F, Imaeda T 1971 Interaction of Pseudomonas bacteriophage 2 with the slime polysaccharide and lipopolysaccharide of Pseudomonas aeruginosa strain BI. Journal of Virology 8:311-317.

Belcher R, Nutten A J, Sambrook C M 1954 The determination of glucosamine. Analyst 79:201-208.

Bitter T, Muir H M 1962 A modified uronic acid carbazole reaction. Analytical Biochemistry 4:330-334.

Brown M R W, Richards R M E 1964 Effect of polysorbate (tween) 80 on the resistance of Pseudomonas aeruginosa to chemical inactivation. Journal of Pharmacy and Pharmacology 16: Supplement 51T-55T.

Bryan L E, Van Den Elzen H M 1977 Spectrum of antibiotic resistance in clincal isolates of Pseudomonas aeruginosa In: Schlessinger D (ed) Microbiology 1977. American Society for Microbiology. Washington, D.C. pp 164-168.

Chen P S, Toribara T Y, Warner H 1956 Microdetermination of phosphorus. Analytical Chemistry 28:1756-1758.

Dimitracopoulos G, Bartell P F 1979 Phage-related surface modifications of Pseudomonas aeruginosa: Effects on the biological activity of viable cells. Infection and Immunity 23:87-93.

Dimitracopoulos G, Bartell P F 1980 Slime glycolipoproteins and the pathogenicity of various strains of Pseudomonas aeruginosa in experimental infection. Infection and Immunity 30:402-408.

Dimitracopoulos G, Intzes C, Papavassiliou J 1979 Characteristics of Pseudomonas aeruginosa in relation to laboratoryinduced resistance to gentamicin. Journal of Clinical Patho$\log y$ 32: $723-727$.

Ellwood D C 1970 The distribution of 2-keto-3-deoxy-octonic acid in bacterial walls. Journal of General Microbiology 60:373-380.

Haynes W C 1951 Pseudomonas aeruginosa-its characterization and identification. Journal of General Microbiology 5:939950 .
Lambris J, Papamichail M, Ioannidis C, Dimitracopoulos G 1982 Activation of the alternative pathway of human complement by the extracellular slime glycolipoprotein of Pseudomonas aeruginosa. Journal of Infectious Diseases 145:78-82.

Lambris J., Papavassiliou J, Dimitracopoulos G 1980 Protein component of Pseudomonas aeruginosa slime glycolipoprotein in relation to laboratory-induced resistance to gentamicin. Canadian Journal of Microbiology 26:852-853.

Lowry O H, Rosebrough N J, Farr A L, Randall R J 1951 Protein measurement with the Folin phenol reagent. Journal of Biological Chemistry 193:265-275.

Lynn M, Sensakovic J W, Bartell P F 1977 In vivo distribution of Pseudomonas aeruginos a slime glycolipoprotein: association with leukocytes. Infection and Immunity 15:109-114.

Osborn M J 1963 Studies on the gram-negative cell wall. I. Evidence for the role of 2-keto-3-deoxyoctonate in the lipopolysaccharide of Salmonella typhimurium. Proceedings of the National Academy of Sciences of the USA. 50:499-506.

Papamichail M, Dimitracopoulos G, Tsokos G, Papavassiliou J 1980 A human lymphocyte mitogen extracted from the extracellular slime layer of Pseudomonas aeruginosa. Journal of Infectious Diseases 141:686-688.

Pechey D T, James A M 1973 Surface lipid of cells of Pseudomonas aeruginosa and its relation to gentamicin resistance. Biomedicine 19:127-129.

Reese J F, Dimitracopoulos G, Bartell P F 1974 Factors influencing the adsorption of bacteriophage 2 to cells of Pseudomonas aeruginosa. Journal of Virology 13:22-27.

Sensakovic J W, Bartell P F 1974 The slime of Pseudomonas aeruginosa: Biological characterization and possible role in experimental infection. Journal of Infectious Diseases 129:101-109.

Spiro R G 1966 Analysis of sugars found in glycoproteins. Methods in Enzymology 8:3-26.

Sykes R B, Morris A 1975 Resistance of Pseudomonas aeruginosa to anti-microbial drugs. p. 333-393. In: Ellis G P, West G B (eds) Progress in Medicinal Chemistry, vol 12. North Holland, Amsterdam, pp 333-393. 